Research Article

\title{
Genomic variation and population structure detected by single nucleotide polymorphism arrays in Corriedale, Merino and Creole sheep
}

Andrés N. Grasso ${ }^{1}$, Virginia Goldberg ${ }^{1}$, Elly A. Navajas ${ }^{2}$, Wanda Iriarte ${ }^{2}$, Diego Gimeno ${ }^{3}$, Ignacio Aguilar ${ }^{1}$, Juan F. Medrano ${ }^{4}$, Gonzalo Rincón ${ }^{4}$ and Gabriel Ciappesoni ${ }^{1}$

${ }^{1}$ Meat and Wool National Program, Instituto Nacional de Investigación Agropecuaria, Las Brujas, Canelones, Uruguay.

${ }^{2}$ Biotechnology Unit, Instituto Nacional de Investigación Agropecuaria, Las Brujas, Canelones, Uruguay.

${ }^{3}$ Department of Animal Genetic Improvement, Secretariado Uruguayo de la Lana, Montevideo, Uruguay.

${ }^{4}$ Department of Animal Science, University of California Davis, USA.

\begin{abstract}
The aim of this study was to investigate the genetic diversity within and among three breeds of sheep: Corriedale, Merino and Creole. Sheep from the three breeds (Merino $n=110$, Corriedale $n=108$ and Creole $n=10$ ) were genotyped using the Illumina Ovine SNP50 beadchip ${ }^{\oplus}$. Genetic diversity was evaluated by comparing the minor allele frequency (MAF) among breeds. Population structure and genetic differentiation were assessed using STRUCTURE software, principal component analysis $(P C A)$ and fixation index $\left(F_{S T}\right)$. Fixed markers $(M A F=0)$ that were different among breeds were identified as specific breed markers. Using a subset of 18,181 single nucleotide polymorphisms (SNPs), PCA and STUCTURE analysis were able to explain population stratification within breeds. Merino and Corriedale divergent lines showed high levels of polymorphism ( $89.4 \%$ and $86 \%$ of polymorphic SNPs, respectively) and moderate genetic differentiation $\left(\mathrm{F}_{\mathrm{ST}}=0.08\right)$ between them. In contrast, Creole had only $69 \%$ polymorphic SNPs and showed greater genetic differentiation from the other two breeds ( $F_{\mathrm{ST}}=0.17$ for both breeds). Hence, a subset of molecular markers present in the OvineSNP50 is informative enough for breed assignment and population structure analysis of commercial and Creole breeds.
\end{abstract}

Keywords: allele frequencies breed composition, fixation index, Ovine SNP50, population stratification, principal component analysis.

Received: August 29, 2013; Accepted: March 16, 2014.

\section{Introduction}

Natural and artificial selection processes in domestic animals occurred over a relatively short period of time $(10,000$ years) (Zeder, 2008), leading to a wide range of phenotypes with large genetic differences between breeds (Kijas et al., 2009). Genomic information and the vast databases of polymorphisms that are currently available provide a unique opportunity to study loci that were under selection between breeds in this recent history (Hayes et al., 2008; Flori et al., 2009). Several genomes have been sequenced and provide the information needed to identify single nucleotide polymorphisms (SNPs) (Ding and Jin, 2009), the most common variations in DNA sequences. The abundance of SNPs, their genome-wide distribution and the availability of a large number of commercial platforms for high-throughput genotyping make SNPs ideal markers for a variety of genomic studies (Ding and Jin, 2009).

Send correspondence to A. N. Grasso. Meat and Wool National Program, Instituto Nacional de Investigación Agropecuaria, Las Brujas, Canelones, $90200 \quad$ Uruguay. E-mail: nicograsso26@gmail.com.
The OvineSNP50 beadchip ${ }^{\circledR}$ (OvineSNP50), which was developed by Illumina in collaboration with the International Sheep Genomics Consortium (ISGC), is a genome-wide genotyping array for the ovine genome. This beadchip contains 54,241 SNPs that were chosen for being uniformly widespread across the ovine genome (with a coverage involving an average gap size and distance of $50.9 \mathrm{~kb}$ and $46 \mathrm{~kb}$, respectively) and for their high levels of polymorphism in the more than 75 economically important sheep breeds in which they were evaluated by the ISGC (OvineSNP50 Datasheet - Illumina 2010).

The information provided by the OvineSNP50 has many applications, including identification of quantitative trait loci, genome-wide association studies (GWAS), characterization of genetic variability among breeds, genomic selection and genetic comparison between breeds (Kijas et al., 2009; Alam et al., 2011). Differences in genotyping results reported among breeds (Kijas et al., 2012) may have unfavourable effects on these applications, e.g., the contribution of population stratification in generating false positive results in GWAS (Kemper et al., 2011). For this reason, many studies have focused on assessing the genetic 
relatedness and substructure within livestock populations in GWAS (Zenger et al., 2007). As part of current efforts to undertake GWAS in sheep populations, the aim of this study was to use the OvineSNP50 beadchip to investigate the genetic diversity and population structure within and among Corriedale, Merino and Creole breeds.

\section{Materials and Methods}

\section{Selected animals}

Data from 228 animals (98 Corriedale and $100 \mathrm{Me}$ rino lambs, and 10 sires of each of the three breeds) were analyzed. The Corriedale lambs were from CIEDAG (Centro de Investigacion Doctor Alberto Gallinal), a research station of the Uruguayan Wool Secretariat (SUL); since 1998 this breed has been bred selectively for resistance/susceptible (R/S) to gastrointestinal parasites (GIP). This genetic selection has been based on Expected Progeny Differences (EPD) for fecal worm egg count (FEC), as an indicator of the genetic merit of R/S to GIP (Castells and Gimeno, 2011). Merino lambs were sampled from the Fine Merino Nucleus maintained at the Tacuarembó Experimental Station of the National Agricultural Research Institute (INIA) of Uruguay. These animals had been selected for wool quality improvement (Montossi et al., 2005).

To minimize the possible influence of relationship in the dataset, Merino and Corriedalelambs with an average relatedness coefficient value $(\mathrm{AR})<0.04$ were selected. The AR values were calculated using ENDOG v. 4.6 software (Gutiérrez and Goyache, 2005) based on the pedigree of Merino and Corrriedale. Similarly, 10 Corriedale and 10 Merino sires that were unrelated for at least two generations were genotyped. The second selection criterion was the occurrence of extreme values for the FEC of EPD for these two breeds. Finally, 10 Creole sires were selected from the San Miguel National Park in Rocha, Uruguay. Since there are no pedigree records for the Creole flock, the selection criterion was based on maximizing the observed phenotypic variation (wool colour and presence of one or two pairs of horns).

\section{DNA extraction, genotyping and quality control}

DNA was isolated from blood samples (Medrano et $a l ., 1990)$ and then genotyped in GeneSeek (Lincoln, NE) using the OvineSNP50 ${ }^{\circledR}$ beadchip (Illumina, San Diego, CA). Samples with call rates $<80 \%$ were not considered for further analysis. Likewise, SNPs with call rates $<90 \%$ were excluded. Only autosomal markers were evaluated and Map OAR v. 2.0 was used in this study.

\section{Genetic diversity}

Basic indices of genetic diversity, including the percentages of polymorphic markers, observed heterozygosity and expected heterozygosity, were calculated using all autosomal markers in conjunction with GoldenHelix SNP \& Variation Suite (SVS) software (GoldenHelix Inc., Bozeman, MT, USA). For each group, the number of animals (n) with a call rate $>80 \%$, the number of autosomal SNPs with a call rate $>90 \%$ and the average observed heterozygosity $(\mathrm{Ho})$ and expected heterozygosity $(\mathrm{He})$ were calculated using all SNPs, based on the observed genotype frequencies. All of this information is presented in Table 1.

Allele frequencies were calculated with Golden Helix SVS software for each breed and used to assess the Ovine SNP50 performance. Rare and fixed alleles were determined by the minor allele frequency (MAF), with $\mathrm{MAF}<0.01$ and equal to 0 , respectively. Highly polymorphic alleles were defined as those with MAF values between 0.3 and 0.5 . A comparative analysis was used to identity which SNPs were fixed or polymorphic in the genome of each breed.

\section{Genetic population structure}

The subset of autosomal SNPs used in these analysis (STRUCTURE, principal component analysis -PCA and the fixation index $-\mathrm{F}_{\mathrm{ST}}$ ) for each breed were in moderate linkage disequilibrium $\left(\mathrm{r}^{2}<0.4\right.$, with MAF $\left.>0.01\right)$, were not in Hardy-Weinberg equilibrium and were common to the three breeds. These criteria were applied to identify informative molecular markers for each breed.

Subdivisions between and within the populations were evaluated using the linkage model of STRUCTURE software version 2.3.3 (Pritchard et al., 2000), with the number of clusters $(\mathrm{K})$ ranging from two to nine. Ten runs of 10,000 iterations after a burn-in of 10,000 iterations were performed for each $\mathrm{K}$. The most appropriate $\mathrm{K}$ was identified by the $\Delta \mathrm{K}$ method (Evanno et al., 2005). Population structure was also inferred by PCA of the marker data (Patterson et al., 2006). The genetic differentiation between breeds was calculated using the $\mathrm{F}_{\mathrm{ST}}$ (Weir and Cockerham, 1984), which assesses the reduction in genotypic heterozygosity. This parameter can range from zero (no genetic divergence between the subpopulations or from the ancestral population) to one (complete isolation of the subpopulations from each other and from the overall population). Moderate and large differentiations had $\mathrm{F}_{\mathrm{ST}}$ values ranging from 0.05 to 0.15 and 0.15 to 0.25 , respectively. The PCA, $\mathrm{F}_{\mathrm{ST}}$ and marker filters were calculated using Golden Helix SVS software.

Table 1 - Number of samples (n) with a call rate $(\mathrm{CR})>80 \%$, number of autosomal SNPs with a call rate $>90 \%$ (out of a total of 52,413 ) and observed (Ho) and excepted (He) heterozygosity for each breed.

\begin{tabular}{lcccc}
\hline Sheep breed & $\mathrm{n}$ & SNP with CR $>90 \%$ & Ho & He \\
\hline Corriedale & 107 & 48,862 & 0.3549 & 0.3549 \\
Merino & 104 & 51,287 & 0.3772 & 0.3618 \\
Creole & 10 & 49,955 & 0.2852 & 0.2580 \\
\hline
\end{tabular}




\section{Results and Discussion}

\section{Genotypes and quality control}

Most samples $(96.9 \%$ ) had call rates $>80 \%$ (only seven samples had call rates $<80 \%$ and were therefore excluded from the analysis). Overall, 107 Corriedale, 104 Merino and 10 Creole samples were used for this study. Despite the differences among the three breeds, our results showed that OvineSNP50 had a coverage similar to that expected in the design of the beadchip (average gap size of $50.9 \mathrm{~kb}$, OvineSNP50 Datasheet - Illumina, 2010), as well as a good genotyping quality as shown by the high sample and marker call rates (Table 1). Although neither Corriedale nor Creole were included among the genotyped breeds in the original beadchip validation (OvineSNP50 Datasheet - Illumina, 2010), the results obtained here were promising.

Not all SNPs present in databases have been validated and their level of polymorphism in different sheep breeds is unknown. However, in this study, the OvineSNP50 showed similar coverage for the three breeds (one SNP per 50.67 $\mathrm{kb}, 50.77 \mathrm{~kb}$ and $50.76 \mathrm{~kb}$ for Corriedale, Creole and Merino, respectively).Table 1 shows the three indices of genetic diversity that were calculated for each population. Based on the observed heterozygosity and expected heterozygosity, the Merino and Corriedale populations were more diverse than Creole. The elevated diversity of Merino and Corriedale animals was also seen in the distribution of minor allele frequencies. As shown in Table 2, Creole animals had an excess of low allele frequency SNPs $(<0.01)$ compared with the other two breeds $(>0.30)$.

\section{Fixed SNPs}

The number of fixed markers varied among breeds with 874, 1449 and 13790 SNPs for Corriedale, Merino and Creole sheep, respectively. Ninety-three percent of Corriedale SNPs with MAF $=0$ were shared with Creole sheep and $69 \%$ with Merino sheep. In Merino, $90 \%$ of the fixed molecular markers were also fixed in Creole sheep. Comparison of the three breeds showed that they shared 585 fixed SNPs (Figure 1). Willing et al. (2012) tested the behavior of three estimators that infer $\mathrm{F}_{\mathrm{ST}}$ and that are commonly used in population genetic studies. Their results showed that population sample size can be significantly reduced (to as small as $n=4-6$ ) by using an appropriate estimator and a large number of bi-allelic genetic markers $(>1,000)$. Thus, conservation genetic studies can yield almost the same statistical power as studies done on model organisms using markers developed with next-generation sequencing.

The Venn diagram in Figure 1 shows common and unique SNPs among the three breeds. The number of exclusive (fixed) SNPs was 99, 99 and 11,190 for Corriedale, Merino and Creole, respectively. These molecular markers could be useful when assigning breed identity or assessing the proportion of a certain breed in a given animal. Wiggans et al. (2010) reported that a set of 622 SNPs is being used to determine breed identity as part of the quality control process for the USDA genotype database for dairy cattle $(\sim 200$

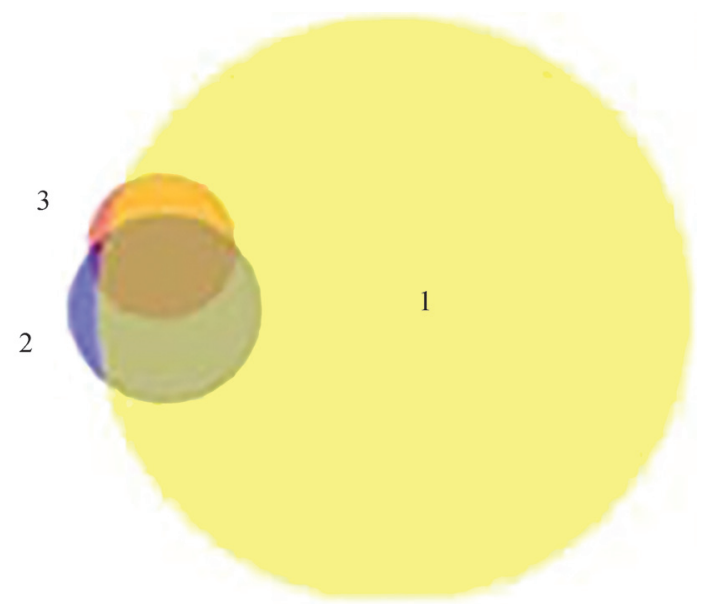

Figure 1 - Venn diagram showing fixed SNPs $(M A F=0)$ in Uruguayan Creole (1), Merino (2) and Corriedale (3) sheep.

Table 2 - Minor allele frequency (MAF) of Corriedale, Merino and Creole sheep breeds. The frequencies refer to autosomal SNPs shared by the three breeds. Min and Max are the minimum and maximum MAF values, respectively. SD -standard deviation.

\begin{tabular}{|c|c|c|c|c|c|c|c|c|}
\hline \multirow[b]{2}{*}{ Breed } & \multirow[b]{2}{*}{ Alleles } & \multicolumn{7}{|c|}{ MAF } \\
\hline & & Min & $\operatorname{Max}$ & Median & Mean & Variance & SD & SNPs \\
\hline \multirow[t]{3}{*}{ Corriedale } & All & 0.00 & 0.50 & 0.28 & 0.27 & 0.02 & 0.14 & 48,862 \\
\hline & Rare & 0.00 & 0.01 & 0.00 & 0.00 & 0.00 & 0.00 & 2,150 \\
\hline & Highly polymorphic & 0.30 & 0.50 & 0.40 & 0.40 & 0.00 & 0.05 & 24,484 \\
\hline \multirow[t]{3}{*}{ Merino } & All & 0.00 & 0.50 & 0.29 & 0.27 & 0.02 & 0.14 & 49,955 \\
\hline & Rare & 0.00 & 0.01 & 0.00 & 0.00 & 0.00 & 0.00 & 1,715 \\
\hline & Highly polymorphic & 0.30 & 0.50 & 0.40 & 0.40 & 0.00 & 0.05 & 25,449 \\
\hline \multirow[t]{3}{*}{ Creole } & All & 0.00 & 0.50 & 0.20 & 0.19 & 0.02 & 0.16 & 51,287 \\
\hline & Rare & 0.00 & 0.00 & 0.00 & 0.00 & 0.00 & 0.00 & 14,056 \\
\hline & Highly polymorphic & 0.30 & 0.50 & 0.40 & 0.39 & 0.00 & 0.06 & 18,447 \\
\hline
\end{tabular}


monomorphic SNPs for each breed). In pigs, Ramos et al. (2011) sought for breed-specific SNPs in five breeds (Duroc, Landrace, Large White, Pietrain and Wild Boar). In this case, a breed-specific marker was defined as a SNP for which one of the alleles was detected only in one breed (a fixed SNP). One hundred and ninety-three SNPs from the PorcineSNP60 beadchip were found to be breedspecific and then validated using 490 animals of the five breeds (Ramos et al., 2011). Four breed assignment validation tests were done and the results indicated that for all methods tested, $99 \%$ of the animals were correctly assigned. In an analogous manner, the fixed SNPs found in this study to be breed-specific for Corriedale, Merino and Creole sheep could be used for breed identification after validation in an independent group of animals.

\section{Frequencies of polymorphic and very polymorphic SNPS}

The percentage of polymorphic SNPs (MAF > 0.01) present in Merino and Corriedale breeds was $89.4 \%$ and $86 \%$, respectively (Table 2 ). However, only $69 \%$ of the SNPs were polymorphic in Creole sheep. Highly polymorphic SNPs (MAF > 0.3) showed a similar trend to that polymorphic SNPs in the three breeds, with Corriedale and Merino animals having $50.1 \%$ and $50.9 \%$ of highly polymorphic SNPs, respectively; Creole sheep had a lower number of highly polymorphic SNPs than the other breeds (36\%; Table 2).

Corriedale and Merino breeds generally had high levels of polymorphism. This result for Merino sheep was expected because this breed was included in the design/validation of OvineSNP50. Moreover, the results obtained for Merino and Corriedale (Table 2) were similar to those observed in the six breeds (Awassi, Merino, Poll Dorset, Romney, Scottish Blackface and Texel) sequenced during the development of OvineSNP50 (Gutiérrez-Gil et al., 2012). The 50 Merino animals that were used in the beadchip design showed $1.3 \%$ and $48.5 \%$ of rare and highly polymorphic SNPs, respectively. The percentages of highly polymorphic and rare SNPs in the other commercial breeds ranged from $37.8 \%$ to $47.1 \%$ and $3.2 \%$ to $13.9 \%$, respectively (Gutiérrez-Gil et al., 2012), thus supporting the findings described here. Nevertheless, there were more rare SNPs in Uruguayan Merino than in the Merino subsample analyzed by Gutiérrez-Gil et al. (2012). Although not directly considered in the design of OvineSNP50, Corriedale sheep represent a composite breed that resulted from cross breeding between Merino and Lincoln (Mendoza, 1968). Analysis with OvineSNP50 has also revealed high levels of polymorphism in four Spanish breeds, with 95.6-98.5\% of the SNPs being polymorphic and $46.5-50.6 \%$ being highly polymorphic (Gutiérrez-Gil et al., 2012). These values are slightly higher than those found in Merino and Corriedale, probably because the analysis included all SNPs rather than just autosomal SNPs.
The animals used in the present study were selected using the minimum average relatedness (AR) as a selection criterion, and this may have contributed to the high percentage of polymorphic markers by avoiding highly related and inbred animals. The AR may be a useful tool when designing GWAS in order to maximize the genomic information content and reduce the risk of spurious associations.

Creole sheep had significantly lower percentages of polymorphic and very polymorphic SNPs. The lower level of polymorphism in this case could be explained by the small size of the population to which these sheep belong $(\mathrm{n}<200)$ and the small sample of Creole animals with genomic information. In addition, unknown events in the history of this Creole population, such as a severe population bottleneck, could also have contributed to a lower percentage of polymorphic SNPs.

In summary, the levels of polymorphism in the molecular markers screened with OvineSNP50 would be sufficient for GWAS and genomic selection in local commercial breeds and Creole sheep. Many GWAS in sheep based on a similar number of informative SNPs have been reported recently (Becker et al., 2010; Johnston et al., 2011; Zhao et al., 2011; White et al., 2012; Riggio et al., 2013; Våge et al., 2013). Johnston et al. (2011) conducted a GWAS in non-commercial Soay sheep using 36,000 SNPs from Ovine SNP50 and identified more SNPs in and around the Horn gene (RXFP2) that were useful in understanding the genetic basis of this trait. A total of 49,233 SNPs were used in a multi-breed GWAS to identify genomic regions associated with susceptibility to and control of ovine lentivirus (White et al., 2012), although the number of informative SNPs was variable among breeds (Rambouillet 50,275; Polypay 50,264; Columbia 44,258). Significant findings on the genetics of litter size in sheep were reported by Våge $e t$ al. (2013), who included 47,986 SNPs in their analysis (after allowing for 5\% missing data per SNP and 1\% MAF). These authors found a missense mutation in Growth Differentiation Factor 9 that was strongly associated with the number of lambs born in Norwegian White Sheep.

\section{Population structure}

A total of 18,181 autosomal SNPs were present in the three breeds and were informative for the analysis of population structure (Table 3). Figure 2A-C shows the results for population structure provided by STRUCTURE and PCA. Figure 2A shows the clusters provided by STRUCTURE based on Bayesian methods, assuming four hypothetical populations $(\mathrm{K}=4)$ determined by the $\Delta \mathrm{K}$ method (Figure $2 \mathrm{~B}$ ). For $\mathrm{K}=4$, the three breeds were clearly differentiated. Creole animals formed a central cluster (Figure 2A) where as Merino sheep were represented by one main cluster with low levels of admixture, and Corriedale individuals formed two clusters with high levels of admixture.

STRUCTURE methods allow the assignment of individuals to groups based on their genetic similarities, 
Table 3 - Subsets of the 52,413 autosomal SNPs per breed that met the established criteria for the population structure analysis.

\begin{tabular}{lccc}
\hline & Corriedale & Creole & Merino \\
\hline MAF $>0.01$ & 50,418 & 38,623 & 50,950 \\
$\mathrm{CR}>0.9$, MAF $>0.01$ & 46,696 & 37,725 & 48,533 \\
$\mathrm{HW}$ & 52,327 & 51,287 & 51,730 \\
$\mathrm{r}^{2}<0.4$ & 33,595 & 15,196 & 38,806 \\
Total & 30,761 & 37,725 & 35,999 \\
Subset of SNPs & & 18,181 & \\
\hline
\end{tabular}

MAF - minor allele frequency, CR - SNP call rate, HW - Hardy-Weinberg equilibrium, $\mathrm{r}^{2}$ - linkage disequilibrium.

thereby providing information about the number of ancestral populations underlying the observed genetic diversity. Indeed, the representative clusters in Corriedale were also present in Merino with a low ancestry coefficient.A similar pattern was observed in Corriedale, which contained some features of the Merino cluster. The two clusters formed by Corriedale were suggestive of genetic substructuring that resulted from using Corridale animals belonging to divergent selection lines.

Figure $2 \mathrm{C}$ shows the results obtained by PCA. The three breeds were clearly differentiated by the first two principal components that were sufficient to account for the observed population structure, with findings very similar to those provided by STRUCTURE (Figure 2A). Creole sheep were very tightly clustered, whereas Merino breed was located in a separate, looser cluster. PCA analysis also showed division of the Corriedale cluster into two subpopulations.

Genetic relatedness and substructure within populations should be taken into account in order to avoid spurious associations in GWAS (Zenger et al., 2007). The likelihood of false positive associations can be minimized by correction through population stratification (Lander and Schork, 1994) and the removal of outliers. The results of this study indicate that OvineSNP50 provides useful information for identifying population structures and outliers. Both PCA and STRUCTURE yielded two clusters for animals of the same breed that belonged to divergent selection lines. In addition, one animal that had been wrongly assigned to a breed was clearly detected by both methods using the selected informative SNPs.

Genetic differences in population structure and the variation detected in allele frequency could be explained by the fact that the Corriedale, Merino and Creole breeds have undergone different selection processes. In particular, the Corriedale flock has been selected for resistance to parasites, whilst the breeding programme of the Merino nucleus has focused on improving wool quality (Montossi et al., 2005). In contrast, Creole sheep have not been under artificial selection since their introduction to Uruguay in the XVIII century (Fernández, 2000). More Creole animals should be examined to confirm the findings reported here.

Moderate genetic differentiation was observed $\left(\mathrm{F}_{\mathrm{ST}}=0.08\right)$ between the two commercial breeds. Low to moderate genetic similarity has also been reported for commercial cattle breeds (Delgado et al., 2011). On the other hand, the degree of differentiation was greater when Creole
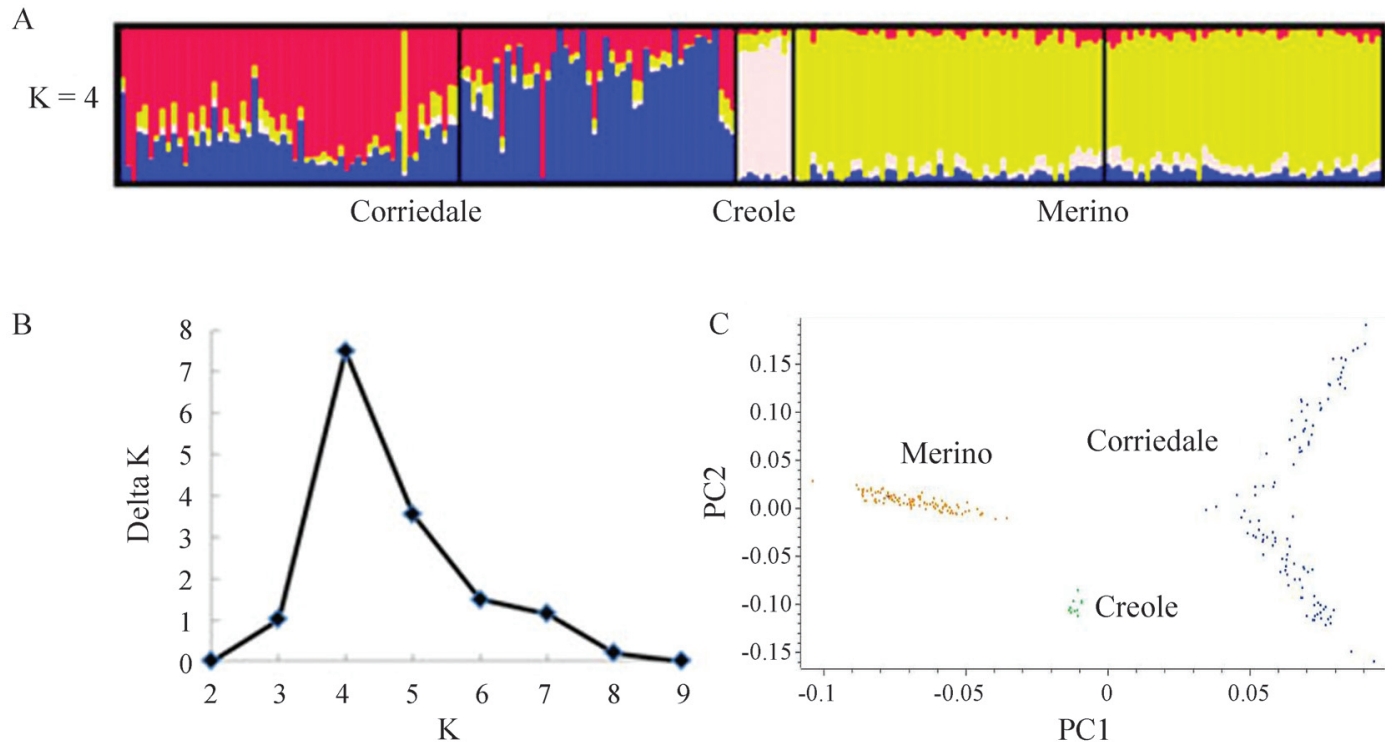

Figure 2 - Population structure analysis estimated for 18,181 SNPs with four clusters $(\mathrm{K}=4)$. (A) Each individual is represented by a vertical line that is divided by $\mathrm{K}$ coloured segments representing the estimated fraction belonging to each cluster. The black borders delimit individuals of different subpopulations. The black lines in the middle of the Merino and Corriedale clusters separate animals resistant and susceptible to gastrointestinal parasites. (B) Delta K plot showing a peak at $\mathrm{K}=4$ (best $\mathrm{K}$ ). (C) First and second principal components. Each point represents one animal and individuals are coloured according to the breed. Each cluster is labelled with the name of the breed. 
Table 4 - Genetic differentiation between breeds, expressed as the fixation index ( $\mathrm{F}_{\mathrm{ST}}$; Weir and Cockerham, 1984) using a subset of 18,181 autosomal SNP frequencies.

\begin{tabular}{lccc}
\hline & \multicolumn{3}{c}{$\mathrm{F}_{\mathrm{ST}}$} \\
\cline { 2 - 4 } & Corriedale & Creole & Merino \\
\hline Corriedale & 0 & & \\
Creole & 0.16 & 0 & \\
Merino & 0.08 & 0.15 & 0 \\
\hline
\end{tabular}

was compared with Corriedale and Merino, with $\mathrm{F}_{\mathrm{ST}}$ values of 0.16 and 0.15 , respectively (Table 4). Thus, both analyses confirmed that Corriedale and Merino were more related to each other than to Creole (Table 4). This finding agrees with the origin of the Corriedale breed, which was derived by crossing Merino and Lincoln sheep 150 years ago (Mendoza et al., 1968). In contrast, Creole has not been under any defined selection process since its introduction to Uruguay in the 1700s (Camacho Vallejo et al., 2000; Fernández, 2000); this 300-year period with no artificial selection is more remote than the common origin between Merino and Corriedale.

Analysis of $\mathrm{F}_{\mathrm{ST}}$ between Churra and Merino showed low differentiation $\left(\mathrm{F}_{\mathrm{ST}}=0.023\right)$ when both breeds were compared (Gutiérrez-Gil et al., 2012), as shown here Merino was genetically more distant from Creole. Moradi et al. (2012) found low differentiation between sheep populations, with mean $\mathrm{F}_{\mathrm{ST}}$ values of 0.024 and 0.027 for the Zel-Lori Bakhtiari and HapMap data sets, respectively. These results agreed with a previous study in which 23 domestic breeds and two wild sheep subspecies were considered and the sheep breeds were found to have generally low differentiation (Kijas et al., 2009). Similar findings have also been reported for Creole cattle compared to commercial cattle (Delgado et al., 2011). Kijas et al. (2012) studied 74 breeds from different parts of the world and assessed the genotype of each animal using nearly 50,000 markers. Their results showed that sheep breeds have maintained high levels of genetic diversity and low genetic differentiation.

In conclusion, the high levels of OvineSNP50 polymorphism in Corriedale, Merino and Creole sheep indicated that the SNPs included in this chip could be used to characterize genetic diversity, identify breeds and detect population structure. This information could be useful for developing specific sets of SNPs for breed assignment (traceability) and parentage testing in commercial settings and for conservation programs. However, the results described here need to be validated with a representative number of animals to ensure accurate results prior to use for commercial or other applications.

\section{Acknowledgments}

The authors thank Daniel Castells, DVM (Uruguayan Wool Secretariat) and Fernando Macedo, DVM (Univer- sidad de la República) for their contribution to this study, and the staff at Glencoe Experimental Research Station (INIA) for data collection and flock management.

\section{References}

Alam M, Lee YM, Park BL, Kim JH, Lee SS, Shin HD, Kim KS, Kim NS and Kim JJ (2011) A whole genome association study to detect single nucleotide polymorphisms for body conformation traits in a Hanwoo population. Asian-Aust J Anim Sci 24:322-329.

Becker D, Tetens J, Brunner A, Bürstel D, Ganter M, Kijas J, International Sheep Genomics Consortium and Drögemüller C (2010) Microphthalmia in Texel sheep is associated with a missense mutation in the paired-like homeodomain 3 (PITX3) gene. PLoS One 5:e8689.

Camacho Vallejo ME, Delgado Bermejo JV and Barba Capote CJ (2000) Recursos genéticos de animales domésticos en España: Situación actual y sistemas tradicionales de explotación. Arch Zootec 49:423-430.

Castells D and Gimeno D (2011) Abstract: Selection of Corriedale sheep for resistance or susceptibility to nematode infection in Uruguay. In Proceedings: 23rd International Conference of the World Association for the Advancement of Veterinary Parasitology. (WAVVP). Buenos Aires, Argentina, 2011.

Delgado JV, Martínez AM, Acosta A, Álvarez LA, Armstrong E, Camacho E, et al. (2011) Genetic characterization of LatinAmerican Creole cattle using microsatellite markers. Anim Genet 43:2-10.

Ding C and Jin S (2009) Single Nucleotide Polymorphisms. Human Press, Totowa, 578:245-254.

Evanno G, Regnaut Sand Goudet J (2005) Detecting the number of clusters of individuals using the software STRUCTURE: A simulation study. Mol Ecol 14:2611-2620.

Fernández G (2000) Situation of the local domestic genetic resources in Uruguay. ArchZootec 49:333-340.

Flori L, Fritz S, Jaffrézic F, Boussaha M, Gut I, Heath S, Foulley JLandGautier M (2009) The genome response to artificial selection: A case study in dairy cattle. PLoS One 4:e6595.

Gutiérrez JP and Goyache F (2005) A note on ENDOG: A computer program for analysing pedigree information. J Anim Breed Genet 122:357-360.

Gutiérrez-Gil B, García-Gámez E, Kijas JW, San Primitivo F, de la Fuente LF, Bayón Y, International Sheep Genomics Consortium (ISGC) and Arranz JJ (2012) Abstract: Preliminary analysis of selective sweeps in the Spanish Churra sheep. In Proceedings National Conference of Animal Genetic Improvement, Menorca, Spain), 4/31/2012, p. 23.

Hayes BJ, Lien S, Nilsen H, Olsen HG, Berg P,MacEachern S, Potter S and Meuwissen THE (2008) The origin of selection signatures on bovine chromosome 6. Anim Genet 39:105111.

Johnston SE, McEwan JC, Pickering NK, Kijas JW, Beraldi D, Pilkington JG, Pemberton JM and Slate J (2011) Genome-wide association mapping identifies the genetic basis of discrete and quantitative variation in sexual weaponry in a wild sheep population. Mol Ecol 20:2555-2566.

Kemper K, Emery D, Bishop S, Oddy H, Hayes BJ, Dominik S, Henshall Jand Goddard M (2011) The distribution of SNP marker effects for faecal worm egg count in sheep, and the 
feasibility of using these markers to predict genetic merit for resistance to worm infections. Genet Res 93:203-219.

Kijas JW, Townley D, Dalrymple BP, Heaton MP, Maddox JF, McGrath A, Wilson P, Ingersoll RG, McCulloch R, McWilliam S, et al. (2009) A genome wide survey of SNP variation reveals the genetic structure of sheep breeds. PLoS One 4:e4668.

Kijas JW, Lenstra JA, Hayes B, Boitard S, Porto Neto LR, San Cristobal M, Servin B, McCulloch R, Whan V, Gietzen K, et al. (2012) Genome-wide analysis of the world's sheep breeds reveals high levels of historic mixture and strong recent selection. PLoS Biology 10:e1001258.

Lander ES and Schork NJ (1994) Genetic dissection of complex traits. Science 265:2037-2048.

Medrano JF, Aasen E and Sharrow L (1990) DNA extraction from nucleated red blood cells. Biotechniques 8:43-43.

Mendoza A (1968) Raza Corriedale. In: Ministerio de Ganadería y Agricultura, Uruguay. Curso Básico Teórico Práctico de Lanares y Lana. Comisión Honoraria de Mejoramiento de la Producción Ovina. Ministerio de Ganadería y Agricultura, Uruguay. p. 120-122.

Montossi F, De Barbieri I, Ciappesoni G, Ravagnolo O, De Mattos D, Pérez Jones J, Fros A, Grattarola M, Mederos A and Soares de Lima M (2005) Núcleo fundacional de merino fino y superfino de la Unidad Experimental "Glencoe" INIA Uruguay: Una experiencia innovadora de mejoramiento genético asociativo y participativo. Agrociencia 1:609616.

Moradi MH, Nejati-Javaremi A, Moradi-Shahrbabak M, Dodds KG and McEwan JC (2012) Genomic scan of selective sweeps in thin and fat tail sheep breeds for identifying of candidate regions associated with fat deposition. BMC Genetics 13:e10.

Patterson N, Price AL and Reich D (2006) Population structure and eigenanalysis. PLoS Genet 2:e190.

Pritchard JK, Stephens M and Donnelly P (2000) Inference of population structure using multilocus genotype data. Genetics 155:945-959.

Ramos M, Megens HJ, Crooijmans RPM, Schook LBand Groenen M (2011) Identification of high utility SNPs for population assignment and traceability purposes in the pig using high-throughput sequencing. Anim Genet 42:613-620.

Riggio V, Matika O, Pong-Wong R, Stear MJ and Bishop SC (2013) Genome-wide association and regional heritability mapping to identify loci underlying variation in nematode resistance and body weight in Scottish Blackface lambs. Heredity 110:420-429.

Våge DI, Husdal M, Kent MP, Klemetsdal G and Boman IA (2013)A missense mutation in growth differentiation factor 9 (GDF9) is strongly associated with litter size in sheep. BMC Genetics 14:e1.

Weir BSand Cockerham CC (1984) Estimating F-statistics for the analysis of population structure. Evolution 38:1358-1370.

White SN, Mousel MR, Herrmann-Hoesing LM, Reynolds JO, Leymaster KA, Neibergs HL, Lewis GS and Knowles DP (2012) Genome-wide association identifies multiple genomic regions associated with susceptibility to and control of ovine lentivirus. PLoS One 7:e47829.

Wiggans GR, VanRaden PM, Bacheller LR, Tooker ME, Hutchison JL, Cooper TA and Sonstegard TS (2010) Selection and management of DNA markers for use in genomic evaluation. J Dairy Sci 93:2287-2292.

Willing E-M, Dreyer C and van Oosterhout C (2012) Estimates of genetic differentiation measured by FST do not necessarily require large sample sizes whenusing many SNP markers. PLoS One 7:e42649.

Zeder MA(2008) Domestication and early agriculture in the Mediterranean Basin: Origins, diffusion, and impact. Proc Natl Acad Sci USA 105:11597-11604.

Zenger KR, Khatkar MS, Cavanagh JA, Hawken RJand Raadsma HW (2007) Genome-wide genetic diversity of Holstein Friesian cattle reveals new insights into Australian and global population variability, including impact of selection. Anim Genet 38:7-14.

Zhao X, Dittmer KE, Blair HT, Thompson KG, Rothschild MF and Garrick DJ (2011) A novel nonsense mutation in the DMP1 gene identified by a genome-wide association study is responsible for inherited rickets in Corriedale sheep. PLoS One 6:e21739.

\section{Internet Resources}

Ovine SNP50 Datasheet - Illumina. 2010. OvineSNP50 DNA analysis kit,http://res.illumina.com/documents/products/datasheets/datasheet_ovinesnp50.pdf. (accessed on May 12, 2012).

Associate Editor: Alexandre Rodrigues Caetano

License information: This is an open-access article distributed under the terms of the Creative Commons Attribution License, which permits unrestricted use, distribution, and reproduction in any medium, provided the original work is properly cited. 